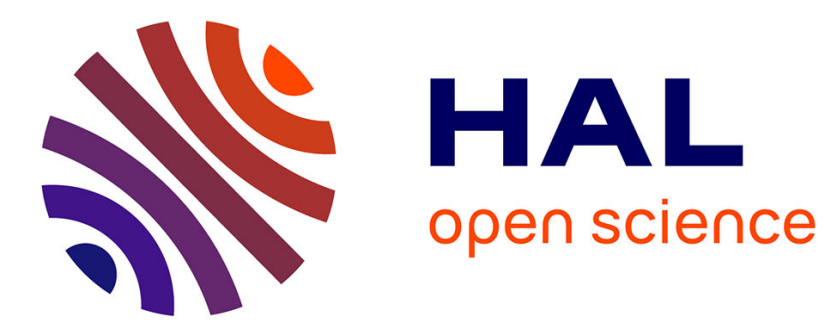

\title{
Ray-traced collision detection for deformable bodies
}

\author{
Everton Hermann, François Faure, Bruno Raffin
}

\section{To cite this version:}

Everton Hermann, François Faure, Bruno Raffin. Ray-traced collision detection for deformable bodies. GRAPP 2008 - 3rd International Conference on Computer Graphics Theory and Applications, Jan 2008, Madeira, Portugal. pp.293-299. inria-00319404v2

\section{HAL Id: inria-00319404 https://hal.inria.fr/inria-00319404v2}

Submitted on 15 Jul 2010

HAL is a multi-disciplinary open access archive for the deposit and dissemination of scientific research documents, whether they are published or not. The documents may come from teaching and research institutions in France or abroad, or from public or private research centers.
L'archive ouverte pluridisciplinaire HAL, est destinée au dépôt et à la diffusion de documents scientifiques de niveau recherche, publiés ou non, émanant des établissements d'enseignement et de recherche français ou étrangers, des laboratoires publics ou privés. 


\title{
RAY-TRACED COLLISION DETECTION FOR DEFORMABLE BODIES
}

\author{
Everton Hermann \\ INRIA \\ Everton.Hermann@imag.fr \\ François Faure \\ Grenoble Universities \\ Francois.Faure@imag.fr \\ Bruno Raffin \\ INRIA \\ Bruno.Raffin@imag.fr
}

Keywords: Collision Detection, Collision Reponse, Physically Based Animation, Deformable Bodies

Abstract: This paper presents a new approach to collision detection and modeling between deformable volumetric bodies. It allows deep intersections while alleviating the difficulties of distance field update. A ray is shot from each surface vertex in the direction of the inward normal. A collision is detected when the first intersection belongs to an inward surface triangle of another body. A contact force between the vertex and the matching point is then created. Experiments show that this approach is fast and more robust than traditional proximity-based collisions.

\section{INTRODUCTION}

Collision detection and response is one of the major computational tasks in physically based animation. It has thus received considerable attention, and the contributions are too numerous to discuss all of them. While special shapes such as spheres or cubes allow the use of optimized methods, the general case of triangular meshes is much more complex. In the case of rigid objects, the most efficient approaches rely on signed distance fields. Each point of one object (which we call the colliding object) is tested against the distance field of the other (called the collided object). If the point is inside the collided object, the nearest point on the collided surface is found and a constraint between these points is created. The test can also be performed the other way round, by switching the colliding and collided objects. Computing a distance field is a compute-intensive task that is performed once at initialization time for rigid objects, and defined with respect to a local reference frame.

When the objects are deformable, the distance field would have to be recomputed at each time step, making it too complex for real-time applications. The most popular strategy is thus to detect pairs of geometric primitives in close proximity, and to set up constraints to keep them apart. In this approach, the contact points are those having a distance to a geometric primitive of the collided body below a given arbitrary proximity threshold. However, discrete time integration may allow the surfaces to cross each other, and when a primitive of the colliding object goes deeper in the collided object than the proximity threshold, it can not be identified as a contact point. This results in poor collision responses that sometimes leave the bodies in intersection, as illustrated in figure 1.

The problem of surface crossing due to discrete time integration can somehow be alleviated using sophisticated strategies based on collision prediction, given current positions and velocities. However, these methods are complex. Their convergence is unclear and they may require short time steps, while large time steps are preferable for real-time applications. Consequently, they have been mainly applied to offline cloth simulations.

For volumetric objects, a reasonable amount of intersection can be visually acceptable, and a robust contact modeling method would allow us to perform large time steps. GPU-based methods can detect the pixels of a colliding surface inside a collided body, but they do not compute the matching points on the collided surface. This prevents a robust setting of the associated contact constraints.

In this paper, we propose to search the matching point along the inward normal of each colliding 


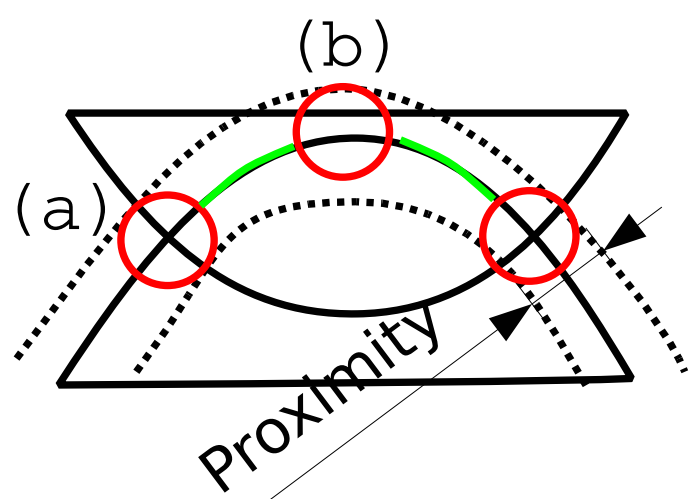

Figure 1: Problems with proximity-based collision detection when the bodies intersect each other. (a): proximities inside and outside the intersection volume may balance each other, resulting in a null net reaction. (b): undesirable contacts may be modeled. Green: large areas of the intersecting surface are ignored.

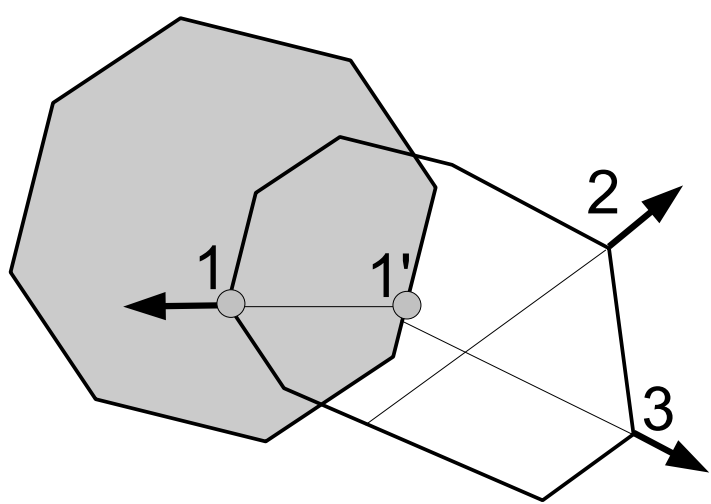

Figure 2: Our ray-traced collision detection. A contact is modeled at points 1 and 1' because the ray shot from point 1 on the colliding object hits the inward surface of the collided object, contrary to points 2 and 3 .

point, using a ray-tracing technique illustrated in figure 2. This approach is simpler than finding the closest primitive, because we search the matching point on a one-dimensional ray, rather than over the whole 3D space. We then apply penalty forces between the matching points. Such forces are necessarily perpendicular to the colliding surface, which ensures a reasonable reaction.

The remainder of this paper is organized as follows. We briefly summarize related work in section 2 . We present our octree-based raytracing in section 3 . Reaction forces are evaluated in section 4 , and results are discussed in section 5 .

\section{RELATED WORK}

The field of collision detection is too large to be reviewed in detail here. An excellent survey of collision detection between deformable objects is given in (Teschner et al., 2005). Bounding volume hierarchies are typically used to accelerate proximity detection (Mezger et al., 2003). Continuous collision detection can be used to avoid intersections in cloth simulation (Bridson et al., 2002). Strangely enough, volumetric elastic bodies have not received much attention. (Guy and Debunne, 2004) apply stochastic proximity-based surface detection, while (Teschner et al., 2003) apply spatial hashing to tetrahedra and apply heuristics to set up contact forces between these elementary volumes. Distance fields (Frisken et al., 2000) are generally used when at least one of the colliding objects is rigid. Their update requires a volumetric mesh and is reduced to small deformations (Fisher and Lin, 2001). Image-space techniques can detect intersections (Heidelberger et al., 2004), but they can only model reactions along the camera axis.

\section{DETECTION}

If we consider a collision pipeline, our algorithm is placed on the Narrow Phase process, as it works with pairs of objects that are potentially colliding. It requires a previous step, the Broad Phase, for identifying pairs of objects whose bounding boxes are colliding. Given such a pair of objects, our algorithm finds pairs of colliding points (one point per object). Collision response forces are then applied to these point pairs.

To identify these pairs of colliding points, we take a vertex on an object surface and follow the opposite direction of the normal up to finding a point in the other object. Our approach allows to solve collisions even if objects are deeply interpenetrated and triangles are not close enough to be detected based on proximity. Also, using the normal give us a good direction to be used with collision reaction penalties, so in the same algorithm we can do the collision detection and the collision reaction.

Once two objects are interpenetrated and colliding points are detected, the collision reaction forces are applied to separate them. Our method does not depend on precomputed data to determine the colliding points. For this reason, it is well suited to deformable objects, where the distance fields are too expensive to be recomputed at each time step.

The search path from one vertex of an object to 
one point on the other object can be represented as a ray with the origin at the vertices and a direction opposite to the normal at the vertex. To speedup the searching of elements that cross this ray, we stored all the triangles of each colliding objects in an octree. Therefore we can easily navigate inside this octree and efficiently find the points crossing the ray. The octree structure allow us to have a satisfying performance independently from the size of the triangles used, which is not the case for a regular grid.

In the next sections, we detail how this octree is built, and how we use this structure to find pairs of colliding points between two objects.

\subsection{Octree Construction}

For each potentially colliding object we create an octree containing the object triangles. We obtain a spatial data structure that allows us to easily find the triangles that intersect a given region. The efficiency of the octree depends on the triangle spatial distribution. One way to construct the octree is to split a cell while it contains more than one triangle. However this approach does not enable to control the number of cells a triangle belongs to.

Our algorithm ensures that each triangle is present in at most eight octree nodes. The goal is to avoid storing a triangle into a large number of octree cells. It ensures a good balance between octree precision and the number of cells to be tested when traversing the octree. To reach that goal, a triangle is stored at the deepest level where cell size is greater than the largest dimension of the triangle's bounding box (see Figure 3). Some triangles can be stored into non leaf nodes, as triangles of different size can be located in the same area.

\subsection{Ray Tracing}

We ray-trace starting only from vertices located in the intersection of the object bounding boxes, which allows us to cull out numerous tests. Our algorithm is decomposed in two phases (see Algorithm 1): search for colliding pairs, and result filtering.

The search phase consists in taking the opposite of the point normal, and following this direction to find a point on the other object. The octree cells are visited using the octree traversal algorithm presented by (Revelles et al., 2000). Each cell of the octree contains a list of triangles that intersect this cell. When a cell is visited, all the triangles it contains are tested against the ray using the algorithm from (Möller and Trumbore, 1997). If an intersection point is found,

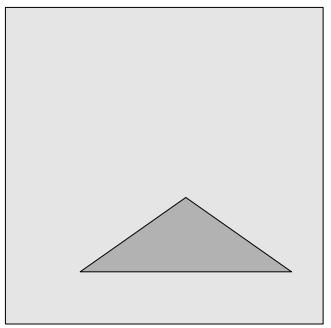

(a)

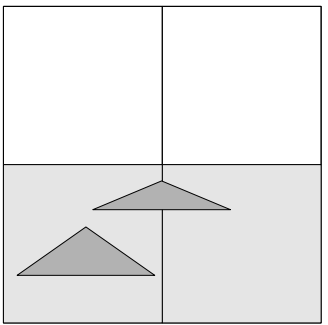

(b)

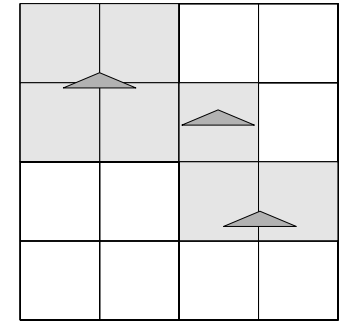

(c)

Figure 3: Quadtree version of the proposed triangle distribution algorithm. (a): a large triangle placed at the first cell level, (b) 2 triangles stored at the second level and (c) 3 triangles at the third level.

this algorithm gives us its coordinates and the distance from the ray's origin.

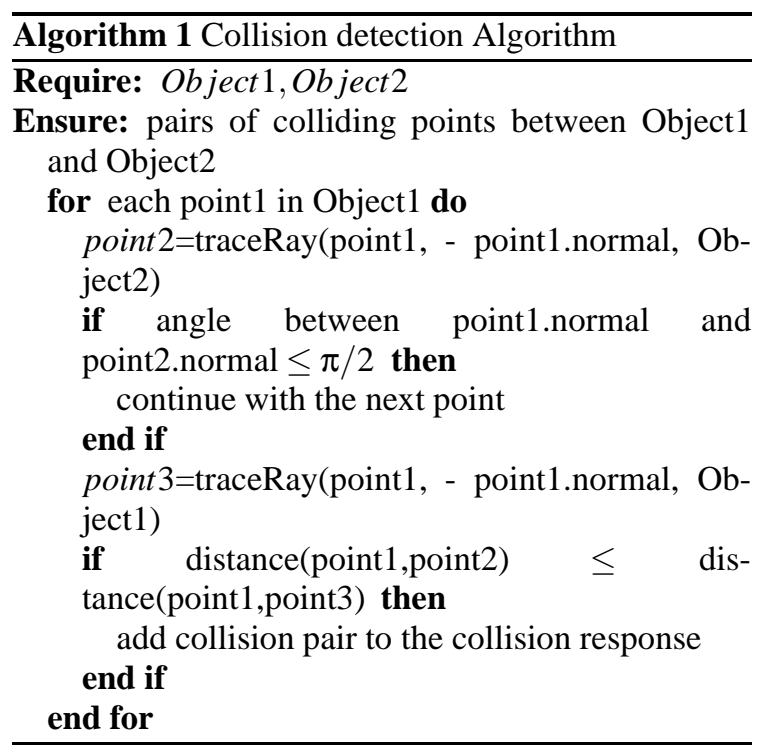

Having a pair of colliding points, one on each object, we test the validity of the resulting contacts as illustrated in Figure 4. The first verification concerns the angle between the normals of both points. An acute angle means that the ray is entering the second object instead of exiting. Eliminating acute angles avoids the misdetection of collision pairs like the one shown in Figure 4(a), where two different rays are 
traced from $\mathrm{O} 1$, but only one is valid, as the angle A is acute. Applying forces to those points would make the objects collide even more.

However, only eliminating colliding pairs that traverse the second object from the outside face is not enough. We must ensure that the point we found is not outside O1, as only using the normal as filter criterion may generate ambiguous results. A point that is part of two triangles can have a normal that satisfies the first criterion even if the point is outside the colliding object. Figure 4(b) illustrates the second validation condition of a colliding point. The ray used to search a colliding point on $\mathrm{O} 2$ is reused to intersect the object $\mathrm{O} 1$. If the point found on $\mathrm{O} 1$ (point3) is closer to the origin of the ray (point1) than the point on $\mathrm{O} 2$, this collision pair is eliminated as the second point is outside object1. The collision pairs that satisfy all the tests are kept to be treated by the collision response phase.

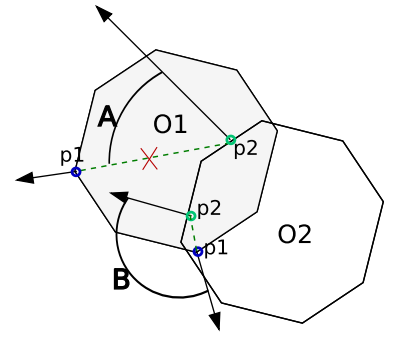

(a)

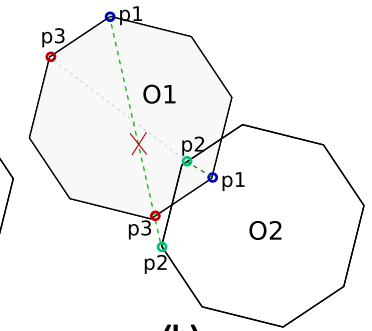

(b)

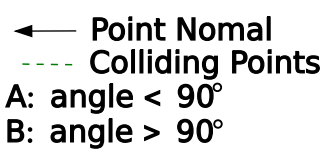

Figure 4: Colliding points validation. Point1, point2 and point 3 denote the points identified by the Algorithm 1 .

\subsection{Self-collision}

Self-collision can be detected using an extension of our method illustrated in Figure 5. A self-collision is detected when the ray hits twice the inward surface of the object. This test is more expensive because all the vertices of a potentially self-intersecting body are tested.

\section{REACTION}

Once collisions are detected and modeled, we apply a penalty force to each pair of matching points. The force is proportional to the distance, and parallel

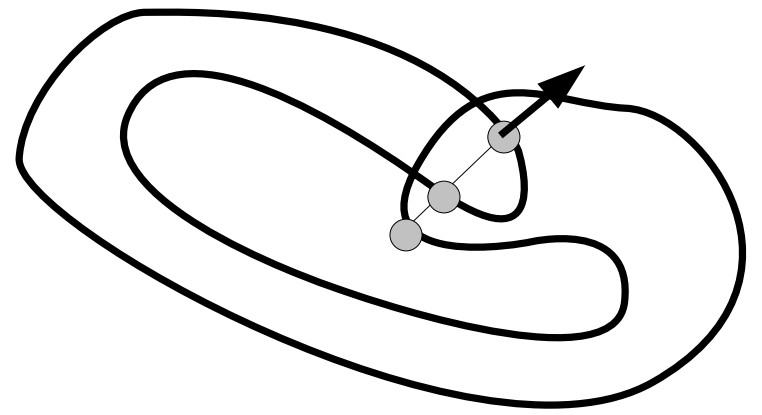

Figure 5: Self-collision detection.

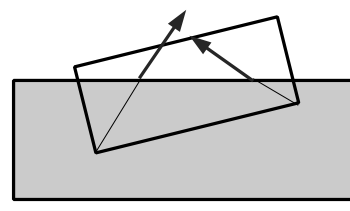

(a)

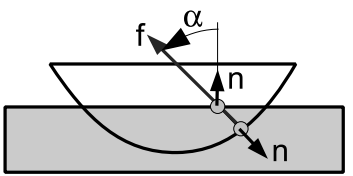

(b)
Figure 6: Contact force. In (a), a sharp object undergoes a non-null net tangential force. In (b), angle $\alpha$ is used to estimate the quality of the contact model and to weight its force.

to the line joining the matching points. This guarantees that Newton's third law on opposite forces and torques is satisfied. We apply the force directly to the colliding point, and we distribute the opposite force to the vertices of the associated triangle in the collided object according to the barycentric coordinates of the intersection point. We perform an implicit time integration to avoid instabilities due to high contact forces.

The direction of the force is not necessarily parallel to the normal of the collided object, as illustrated in Figure 6, and some contact pairs are more reliable than others. As a result, sharp objects may undergo undesirable net tangential forces.

We therefore multiply the intensity of the force by the cosine of the angle $\alpha$ in figure 6(b). This reduces the influence of the less reliable contact forces.

To evaluate the contact forces created by our algorithm, we used a cylinder crossed by a plane, and the expected direction for the resulting force is a vertical force as shown in 2D in Figure 7. Due to symmetry, tangential forces should balance each other and the net tangential force should be null. However, due to surface discretization, as the rotational position of the cylinder changes, the resulting force direction may differ from the normal of the plane.

To measure the variation of the resulting force, we tested cylinders with a number of sides ranging from 

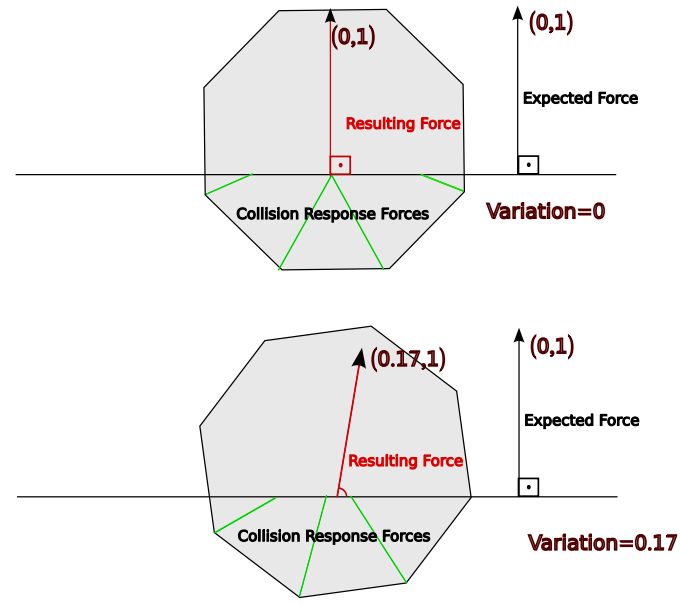

Figure 7: A cylinder undergoing various tangential forces due to low geometric resolution.

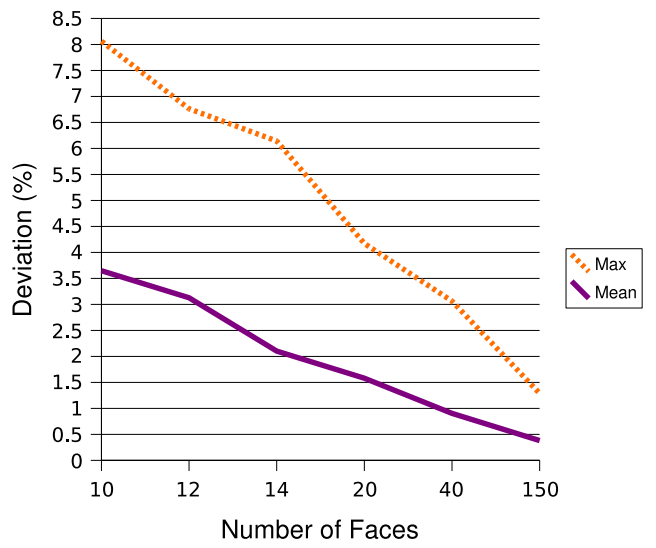

Figure 8: Ratio of tangential and normal force, against the number of cylinder faces.

10 to 150 . For each cylinder we took 100 different rotational positions, and measured the response forces. In Figure 8 we show the mean of the variations for a varying numbers of sides. We observe that the quality of the resulting force increases with the object level of detail. For a cylinder having only 10 sides, we get a worst case deviation of $8 \%$. As we increase the number of sides, variation levels decrease to about $1 \%$. This variation depends of course on the shape of the object, and the results improve with smoother objects.

As our algorithm uses penalties to separate the objects, in some cases we are not able to fully separete them in one step. In those situations using the velocity direction has no meaning, as it was changed by the penalties forces in the previous step. By the way, the usage of the normal direction give us a good aproximation of the collision direction.

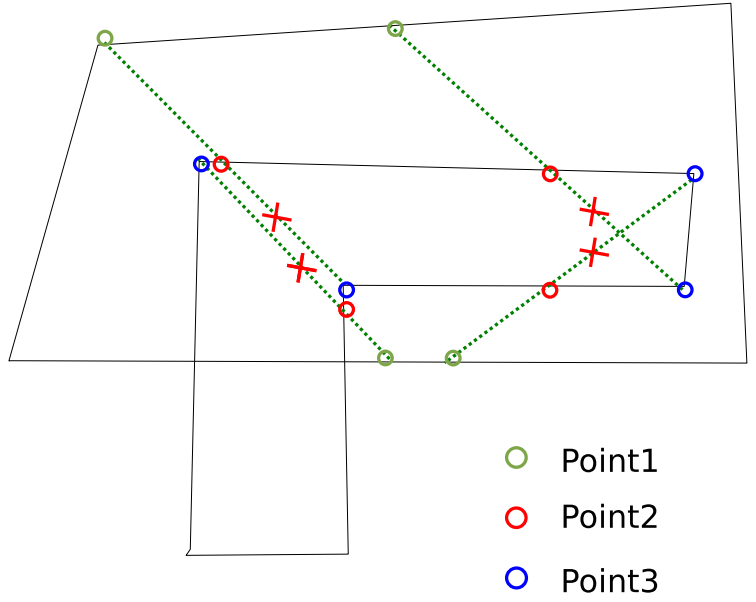

Figure 9: Our method can fail in case of non-convex intersection volume.

A limitation of our methods occurs when all the inward rays cross the colliding body before the collided one, as illustrated in figure 9. In this case, no collision is detected and the contact force is null. Note that this does not induce instabilities. A proximitybased method would succeed if the colliding vertices were not deeper than the proximity threshold, and fail otherwise. The same happens when one object is completly inside the other, and our algorithm does not find a way to separate them.

Another limitation occurs when an object that is in a self-colliding state, also collides another object. In this case, some collision may be missed as the first colliding point may be an outside face. Also edgeedge interpenetration are not always detected as we project the rays from the object vertexes.

\section{RESULTS}

We compared the efficiency of our method with a hierarchical implementation of a proximity-based approach, similar to the one proposed by (Bridson et al., 2002). The algorithms were implemented using the Simulation Open Framework Architecture (SOFA) (Allard et al., 2007). The parallel version of our algorithm was developed using KAAPI (Gautier et al., 2007).

The first test consists in starting the simulation with interpenetrating objects. We observe how the algorithms manage to push the objects apart. Figure 10 shows the starting scene followed by the reaction produced by each algorithm. The ray-tracing algorithm manages to separate the objects, while the objects are only deformed and remain interpenetrated using the 


\section{Initial State}

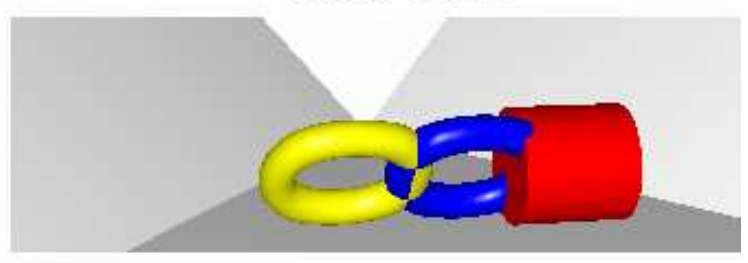

\section{Proximity Response}

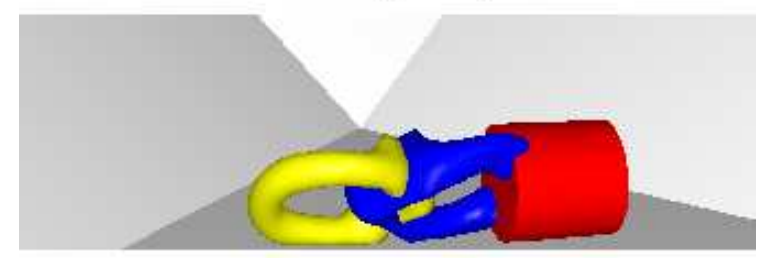

\section{Ray-trace Response}

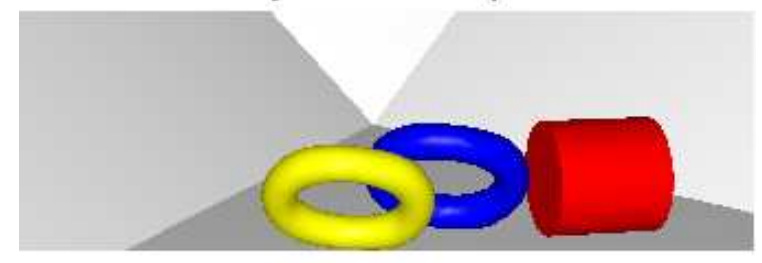

Figure 10: A test scene. Each torus includes 1600 triangles.

proximity-based approach. The ray-tracing applies all the penalties in a direction that separates the objects. The proximity-based algorithm tries just to push apart triangles that are too close. As a result, some penalties are oriented in a direction opposite to the one that should be used to separate the objects.

Another advantage of our approach is the larger simulation step $(d t)$ that can be used. With a large $d t$ objects can move from a non colliding state to a deep interpenetration. With a proximity-based approach, deeply interpenetrated objects lead to triangles too far apart to be detected as colliding. Using the same scene with no initial intersection (Figure 11), the proximity-based algorithm gives satisfying results up to a maximum $d t$ of 0.2 seconds, while our algorithm is still effective up to 0.45 seconds.

In the scene illustrated in Figure 12 we show a similar situation where the interpenetration of objects restrains the movements when using a proximity-

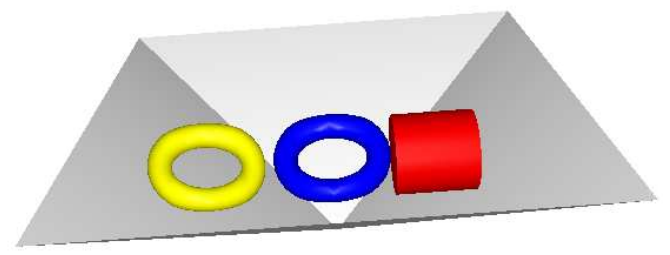

Figure 11: Scene used for performance comparison.

based approach. The rings start the scene with no collision between them, but when they get interpenetrated they are not able to follow the chain movement. However, with the ray trace algorithm the rings can move freely, even with interpenetrations. It allow us tu use larger timesteps without degrading the collision reaction quality.

When running both algorithms on a Xeon $2.5 \mathrm{Ghz}$ machine simulating a scene like the one in Figure 11, our algorithm reaches $30 \mathrm{fps}$, while the proximitybased algorithm reaches only $12 \mathrm{fps}$. It is mainly due to a smaller number of colliding points detected by the ray tracing, as close triangles that are not in a colliding state do not generate colliding points. With less penalties applied, the solver runs faster.

In terms of scalability our algorithm behaves as expected, having a linear degradation of performance as the number of colliding objects increases. The basic element of our collision detection algorithm is the triangle. The algorithm performance depends directly on the number of triangles needing to be evaluated. In Figure 13, we display the time to solve 200 iterations with a varying number of objects in the scene. The objects used are torus initially interpenetrating each other as shown in the attached video.

To take advantage of the available multi-core architecture, we developed a first simple parallel version of the algorithm. Pairs of colliding objects can be computed independently. We take advantage of this parallelism inherent to our algorithm to distribute the pairs to the different processing cores using a work-stealing load balancing strategy. On a quadcore processor, the simulation runs more than twice faster compared to a single core execution. The per- 


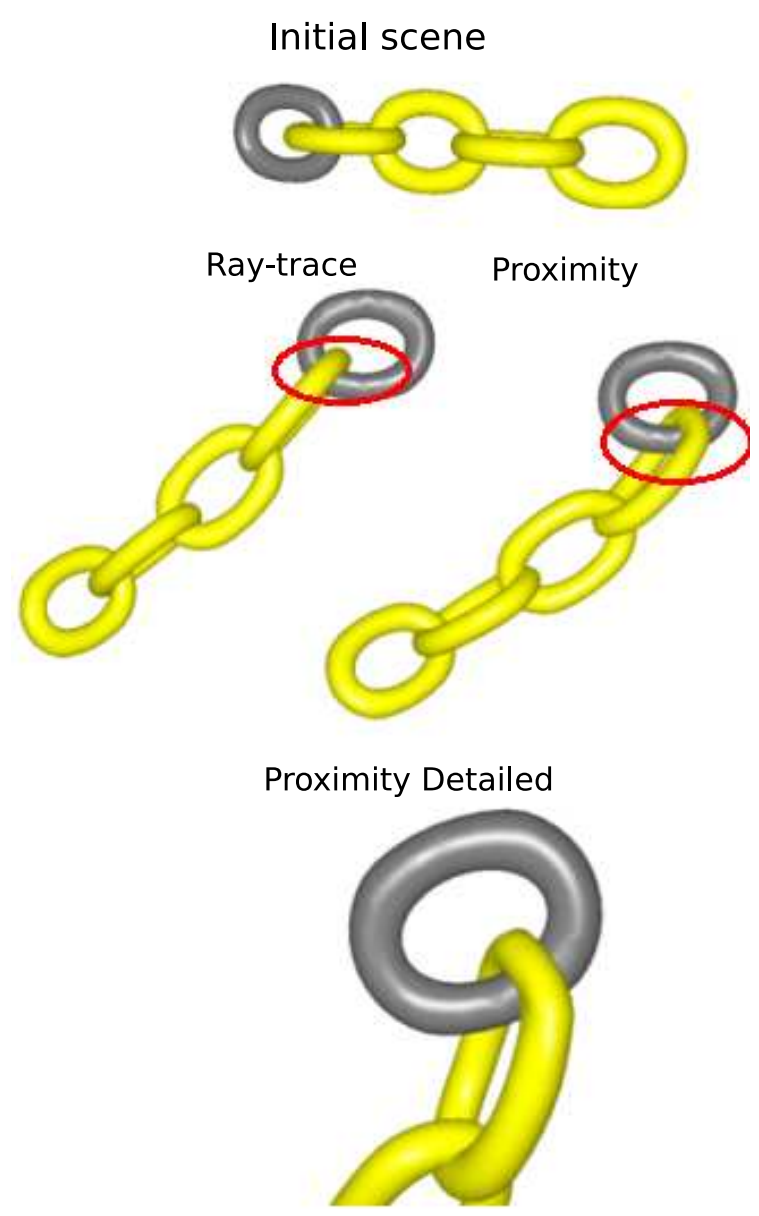

Figure 12: A deformable chain test.

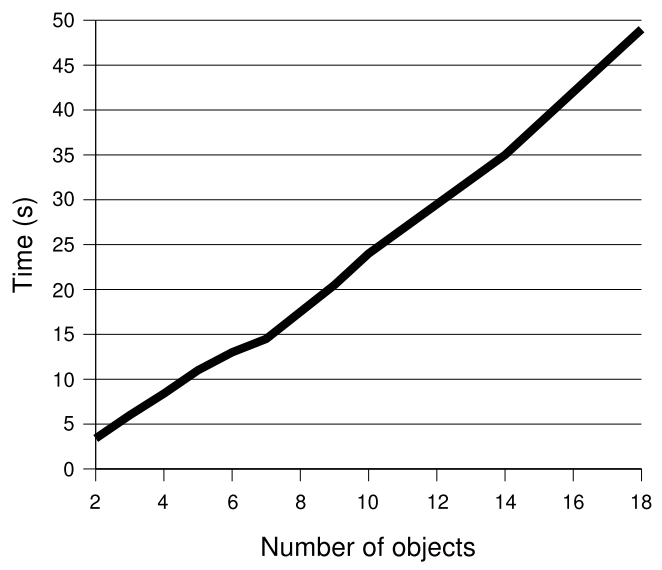

Figure 13: Performance evaluation with a variable number of colliding torus, each of them including 1600 triangles. formance gain is limited by the remaining computations that are sequential.

\section{CONCLUSIONS}

We have shown that our novel collision detection and modeling approach is an interesting alternative to traditional proximity-based methods, especially in the case of smooth deformable volumetric objects. The computation times are shorter, and the robustness allows us to apply larger time steps. The time spent by constructing an octree is compensated by the acceleration obtained on the ray tracing phase.

In future work, we plan to scale the contact force at each colliding vertex by the surface area associated with this vertex, to obtain a more resolutionindependent reaction force. We will also investigate how to cull out more tests in self-intersection detection.

\section{REFERENCES}

Allard, J., Cotin, S., Faure, F., Bensoussan, P.-J., Poyer, F., Duriez, C., Delingette, H., and Grisoni, L. (2007). Sofa - an open source framework for medical simulation. In Medicine Meets Virtual Reality (MMVR'15), Long Beach, USA.

Bridson, R., Fedkiw, R., and Anderson, J. (2002). Robust treatment of collisions, contact and friction for cloth animation.

Fisher, S. and Lin, M. C. (2001). Deformed distance fields for simulation of non-penetrating flexible bodies. In Proceedings of the Eurographic workshop on Computer animation and simulation, pages 99-111, New York, NY, USA. Springer-Verlag New York, Inc.

Frisken, S. F., Perry, R. N., Rockwood, A. P., and Jones, T. R. (2000). Adaptively sampled distance fields: A general representation of shape for computer graphics. In Akeley, K., editor, Siggraph 2000, Computer Graphics Proceedings, pages 249-254. ACM Press / ACM SIGGRAPH / Addison Wesley Longman.

Gautier, T., Besseron, X., and Pigeon, L. (2007). Kaapi: A thread scheduling runtime system for data flow computations on cluster of multi-processors. In PASCO '07: Proceedings of the 2007 international workshop on Parallel symbolic computation, pages 15-23, New York, NY, USA. ACM.

Guy, S. and Debunne, G. (2004). Monte-carlo collision detection. Technical Report RR-5136, INRIA.

Heidelberger, B., Teschner, M., and Gross, M. H. (2004). Detection of collisions and self-collisions using image-space techniques. In WSCG, pages 145152. 
Mezger, J., Kimmerle, S., and Etzmuß, O. (2003). Hierarchical Techniques in Collision Detection for Cloth Animation. Journal of WSCG, 11(2):322-329.

Möller, T. and Trumbore, B. (1997). Fast, minimum storage ray-triangle intersection. Journal of Graphics Tools, 2(1):21-28.

Revelles, J., Ureña, C., and Lastra, M. (2000). An efficient parametric algorithm for octree traversal. In International Conference in Central Europe on Computer Graphics, Visualization and Interactive Media.

Teschner, M., Heidelberger, B., MÃ $\frac{1}{4}$ ller, M., Pomerantes, D., and Gross, M. H. (2003). Optimized spatial hashing for collision detection of deformable objects.

Teschner, M., Kimmerle, S., Heidelberger, B., Zachmann, G., Raghupathi, L., Fuhrmann, A., Cani, M.-P., Faure, F., Magnetat-Thalmann, N., Strasser, W., and Volino, P. (2005). Collision detection for deformable objects. Computer Graphics Forum, 24(1):61-81. 